\title{
INTERACTION OF SHOCK WAVES IN GAS DYNAMICS: UNIFORM IN TIME ASYMPTOTICS
}

\author{
M. G. GARCÍA-ALVARADO, R. FLORES-ESPINOZA, AND G. A. OMEL'YANOV
}

Received 13 May 2005 and in revised form 3 June 2005

We construct a uniform in time asymptotics describing the interaction of two isothermal shock waves with opposite directions of motion. We show that any smooth regularization of the problem implies the realization of the stable scenario of interaction.

\section{Introduction}

We consider the gas dynamics system in the isothermal case

$$
\frac{\partial \rho}{\partial t}+\frac{\partial(\rho u)}{\partial x}=0, \quad x \in \mathbb{R}^{1}, t>0, \quad \frac{\partial(\rho u)}{\partial t}+\frac{\partial}{\partial x}\left(\rho u^{2}+c_{0}^{2} \rho\right)=0,
$$

together with the initial data in the form of two shock waves with opposite directions of motion

$$
\begin{aligned}
& \left.\rho\right|_{t=0}=\rho_{0}+e_{1} H\left(-x+x_{1}^{0}\right)+e_{2} H\left(x-x_{2}^{0}\right), \\
& \left.u\right|_{t=0}=u_{1} H\left(-x+x_{1}^{0}\right)+u_{2} H\left(x-x_{2}^{0}\right) .
\end{aligned}
$$

Here, $H(x)$ is the Heaviside function, $H(x)=1$ for $x>0$, and $H(x)=0$ for $x<0, e_{i}=$ $\rho_{i}-\rho_{0}>0$ are amplitudes of jumps, and $\rho_{i}, u_{i}, c_{0}>0$ are constants. For definiteness, we assume that $\rho_{1} \geqslant \rho_{2}$ and $x_{1}^{0}<x_{2}^{0}$. The initial shock waves are assumed to be stable, so that

$$
\begin{aligned}
& u_{1}=c_{0}\left(\sqrt{\frac{\rho_{1}}{\rho_{0}}}-\sqrt{\frac{\rho_{0}}{\rho_{1}}}\right)>0, \\
& u_{2}=-c_{0}\left(\sqrt{\frac{\rho_{2}}{\rho_{0}}}-\sqrt{\frac{\rho_{0}}{\rho_{2}}}\right)<0 .
\end{aligned}
$$

The solution of problem (1.1), (1.2) seems nowadays to be well known. Indeed, the standard procedure of "step-by-step" consideration before and after the interaction time instant $t=t^{*}$ shows that the solution is described by the two noninteracting shock waves 
3112 Interaction of shock waves in gas dynamics

for $t<t^{*}$, namely,

$$
\begin{aligned}
& \rho=\rho_{0}+e_{1} H\left(-x+\varphi_{10}(t)\right)+e_{2} H\left(x-\varphi_{20}(t)\right), \\
& u=u_{1} H\left(-x+\varphi_{10}(t)\right)+u_{2} H\left(x-\varphi_{20}(t)\right),
\end{aligned}
$$

where $\varphi_{i 0}=\varphi_{i 0_{t}} t+x_{i}^{0}$ are the phases of the shocks,

$$
\varphi_{10_{t}}=u_{1}+c_{0} \sqrt{\frac{\rho_{0}}{\rho_{1}}}=c_{0} \sqrt{\frac{\rho_{1}}{\rho_{0}}}, \quad \varphi_{20_{t}}=u_{2}-c_{0} \sqrt{\frac{\rho_{0}}{\rho_{2}}}=-c_{0} \sqrt{\frac{\rho_{2}}{\rho_{0}}},
$$

and $\varphi_{10}\left(t^{*}\right)=\varphi_{20}\left(t^{*}\right) \stackrel{\text { def }}{=} x^{*}$ is the point of intersection of the paths $x=\varphi_{i 0}(t), i=1,2$.

Next, at the time $t^{*}$, the initial conditions (1.2) are replaced by the shock wave with the amplitudes $\rho_{1}-\rho_{2}$ and $u_{1}-u_{2}$ of the jumps of $\rho$ and $u$, which are concentrated at the point $x=x^{*}$. Solving this Riemann problem, we obtain that the solution for $t>t^{*}$ is again represented by two noninteracting shock waves with uniquely defined new amplitudes and new paths of propagation (see, e.g., $[2,9]$ ). Let us call this behavior of the solution the "stable scenario."

However, the uniqueness of weak solutions for hyperbolic systems of conservation laws has been proved (with additional conditions) only for the case of sufficiently small amplitudes of shocks (see $[1,2,8]$ ). Apart from the above mentioned solution, the Riemann problem admits a family of artificial solutions. Therefore, the described construction cannot be treated as a well-posed one for the case of arbitrary amplitudes of shocks.

It is clear that the weak point of this scheme is the consideration of shock waves as noninteracting ones for time close to $t^{*}$. Moreover, this conflicts with the physical sense of the problem since the actual gas dynamics includes viscosity phenomena. Therefore, it is necessary to smooth the solution for time close to $t^{*}$, and to consider the process of interaction in detail.

Whitham [10] was the first to solve a similar problem for the inviscid Burgers-Hopf equation

$$
\frac{\partial u}{\partial t}+\frac{\partial}{\partial x} f(u)=0
$$

with the quadratic nonlinearity $f(u)=u^{2}$. Passing to the Burgers regularization and using the Hopf-Cole transformation, G. Whitham found the exact solution for the initial data similar to (1.2) and, as a result, established that the regularization implies the choice of a stable scenario of interaction. However, this procedure works uniquely for the quadratic nonlinearity. A progress in this problem has been achieved only recently by Danilov and Shelkovich for (1.6) with convex nonlinearities (see [5]; see also [3, 4]). Since it is impossible to find exact solutions in the general case, they constructed an asymptotic solution in the framework of the "weak asymptotic method" $[3,4,5,6,7]$. The main point here is the treatment of the solution $u_{\varepsilon}(x, t)$ of the regularized problem as a $\mathscr{C}^{\infty}\left(0, T ; \mathscr{C}^{\infty}\left(\mathbb{R}^{1}\right)\right)$ mapping for $\varepsilon=$ const $>0$ and a $\mathscr{C}\left(0, T ; \mathscr{D}^{\prime}\left(\mathbb{R}^{1}\right)\right)$ mapping uniformly in $\varepsilon \in[0,1]$, where $\varepsilon$ is a parameter of regularization. Respectively, a family $u_{\varepsilon}(t, x)$ is called 
an asymptotic $\bmod O_{\mathscr{D}^{\prime}}(\varepsilon)$ solution of $(1.6)$ if the relation

$$
\frac{d}{d t} \int_{-\infty}^{\infty} u_{\varepsilon} \psi d x-\int_{-\infty}^{\infty} f\left(u_{\varepsilon}\right) \frac{\partial \psi}{\partial x} d x=\mathcal{O}(\varepsilon)
$$

holds for any test function $\psi=\psi(x)$. The main advantage of this approach is the possibility to describe the interaction of nonlinear waves by an ordinary differential equation. Let us note that this method allows also to describe soliton interactions for nonintegrable problems $[4,7]$.

Our aim is a generalization of the weak asymptotic method for hyperbolic systems of conservation laws. Using system (1.1) as a simple but meaningful example, we show that this tool easily allows to construct an asymptotic solution. At the same time, we obtain in this way a scattering-type problem for a dynamical system (instead of an equation in the scalar case). Analysis of this problem requires the use of the specifics of the original problem. However, this can be done, and we obtain a uniform in time description of the interaction of two shock waves in the case of opposite directions of motion.

\section{Construction of the asymptotic solution}

Following the ideas sketched above, we arrive at what follows.

Definition 2.1. Sequences $\rho_{\varepsilon}(t, x)$ and $u_{\varepsilon}(t, x)$ are called a weak asymptotic mod $O_{\mathscr{D}^{\prime}}(\varepsilon)$ solution of system $(1.1)$ if $\rho_{\varepsilon}(t, x)$ and $u_{\varepsilon}(t, x)$ belong to $\mathscr{C}^{\infty}\left([0, T] \times \mathbb{R}^{1}\right)$ for $\varepsilon=$ const $>0$ and to $\mathscr{C}\left(0, T ; \mathscr{D}^{\prime}\left(\mathbb{R}^{1}\right)\right)$ uniformly in $\varepsilon \in[0$, const $]$, and if the relations

$$
\begin{gathered}
\frac{d}{d t} \int_{-\infty}^{\infty} \rho_{\varepsilon} \psi_{1} d x-\int_{-\infty}^{\infty} \rho_{\varepsilon} u_{\varepsilon} \frac{\partial \psi_{1}}{\partial x} d x=\mathcal{O}(\varepsilon) \\
\frac{d}{d t} \int_{-\infty}^{\infty} \rho_{\varepsilon} u_{\varepsilon} \psi_{2} d x-\int_{-\infty}^{\infty}\left(\rho_{\varepsilon} u_{\varepsilon}^{2}+c_{0}^{2} \rho_{\varepsilon}\right) \frac{\partial \psi_{2}}{\partial x} d x=\mathcal{O}(\varepsilon)
\end{gathered}
$$

hold for any test functions, $\psi_{i}=\psi_{i}(x) \in \mathscr{D}\left(\mathbb{R}^{1}\right)$.

It is necessary to note that the parabolic regularization of $(1.1)$ with $\mathscr{O}(\varepsilon)$ viscosity terms implies $O(\varepsilon)$ corrections in relations (2.1).

To present the asymptotic solution, let us denote $\omega=\omega(\eta) \in \mathscr{C}^{\infty}\left(\mathbb{R}^{1}\right)$ an auxiliary function such that

$$
\lim _{\eta \rightarrow-\infty} \omega=0, \quad \lim _{\eta \rightarrow \infty} \omega=1 .
$$

For simplicity, we assume that $\omega$ tends to its limiting values at an exponential rate. Moreover, we assume that

$$
\omega_{\eta}^{\prime}>0, \quad \omega(\eta)+\omega(-\eta)=1
$$

Obviously, this implies that $\omega(\eta)-1 / 2$ is an odd function and $\omega((x-\phi) / \varepsilon) \rightarrow H(x-\phi)$ as $\varepsilon \rightarrow 0$. 
3114 Interaction of shock waves in gas dynamics

Now, let us write the weak asymptotic solution for the problem (1.1), (1.2) in the following form:

$$
\begin{aligned}
& \rho_{\varepsilon}=\rho_{0}+e_{1} \omega\left(\frac{-x+\phi_{1}}{\varepsilon}\right)+e_{2} \omega\left(\frac{x-\phi_{2}}{\varepsilon}\right)+r \omega\left(\frac{-x+\phi_{1}}{\varepsilon}\right) \omega\left(\frac{x-\phi_{1}}{\varepsilon}\right), \\
& u_{\varepsilon}=u_{1} \omega\left(\frac{-x+\phi_{1}}{\varepsilon}\right)+u_{2} \omega\left(\frac{x-\phi_{2}}{\varepsilon}\right)+v \omega\left(\frac{-x+\phi_{1}}{\varepsilon}\right) \omega\left(\frac{x-\phi_{1}}{\varepsilon}\right) .
\end{aligned}
$$

Here, the phases $\phi_{i}=\phi_{i}(t, \tau)$ are defined by

$$
\phi_{i}=\varphi_{i 0}(t)+\psi_{0}(t) \varphi_{i 1}(\tau), \quad i=1,2,
$$

where

$$
\psi_{0}(t)=\varphi_{20}(t)-\varphi_{10}(t), \quad \tau=\frac{\psi_{0}(t)}{\varepsilon},
$$

and the phases of noninteracting shock waves, $\varphi_{i 0}$, and $\rho_{0}, e_{i}, u_{i}$ are the same as in (1.4), (1.5).

Thus, $\tau$ plays the role of a "fast" time and for time $t$ before (after) the interaction $\psi_{0}(t)>0$ and $\tau \rightarrow+\infty\left(\psi_{0}(t)<0\right.$ and $\left.\tau \rightarrow-\infty\right)$ as $\varepsilon \rightarrow 0$.

The functions $\varphi_{i 1}=\varphi_{i 1}(\tau), r=r(\tau)$, and $v=v(\tau)$ are assumed to be smooth and such that

$$
\begin{gathered}
\varphi_{i 1} \longrightarrow 0, \quad r \longrightarrow 0, \quad v \longrightarrow 0 \quad \text { as } \tau \longrightarrow+\infty, \\
\varphi_{i 1} \longrightarrow \bar{\varphi}_{i 1}, \quad r \longrightarrow \bar{r}, \quad v \longrightarrow \bar{v} \quad \text { as } \tau \longrightarrow-\infty,
\end{gathered}
$$

where $\bar{\varphi}_{i 1}, \bar{r}$, and $\bar{v}$ are some constants.

The first assumption (2.7) implies that the anzatz (2.4) describes the two noninteracting waves (1.4) before the interaction. In order to describe the behavior of the anzatz after the instant time of interaction, we have to analyze the product $\omega\left(\left(-x+\phi_{1}\right) / \varepsilon\right) \omega((x-$ $\left.\phi_{2}\right) / \varepsilon$ ).

LEMмA 2.2. Under the assumptions mentioned above, the following relations hold:

$$
\begin{aligned}
\omega^{k}\left(\frac{x-\phi_{i}}{\varepsilon}\right)= & H\left(x-\phi_{i}\right)+\varepsilon d_{k} \delta\left(x-\phi_{i}\right)+\mathscr{O}_{\mathscr{D}^{\prime}}\left(\varepsilon^{2}\right), \\
\omega^{k}\left(\frac{-x+\phi_{1}}{\varepsilon}\right) \omega^{\ell}\left(\frac{x-\phi_{2}}{\varepsilon}\right)= & b_{k \ell}\left\{H\left(-x+\phi_{1}\right)-H\left(-x+\phi_{2}\right)\right\} \\
& -\varepsilon\left\{c_{k \ell} \delta\left(x-\phi_{1}\right)+c_{\ell k} \delta\left(x-\phi_{2}\right)\right\}+O_{\mathscr{D}^{\prime}}\left(\varepsilon^{2}\right),
\end{aligned}
$$

where $k, \ell \geqslant 1, d_{k}$ are some constants, $d_{1}=0$ and

$$
\begin{aligned}
& b_{k \ell}=k \int_{-\infty}^{\infty} \omega^{k-1}(\eta) \omega_{\eta}^{\prime}(\eta) \omega^{\ell}(-\sigma-\eta) d \eta, \\
& c_{k \ell}=k \int_{-\infty}^{\infty} \eta \omega^{k-1}(\eta) \omega_{\eta}^{\prime}(\eta) \omega^{\ell}(-\sigma-\eta) d \eta .
\end{aligned}
$$


Here and in what follows

$$
\sigma=\frac{\phi_{2}-\phi_{1}}{\varepsilon}, \quad \omega_{\eta}^{\prime}(\eta)=\frac{d \omega(\eta)}{d \eta}
$$

Proof. Relation (2.9) is almost obvious. Let us only note that the equality $d_{1}=0$ is a direct consequence of the equality in (2.3). Furthermore, considering the left-hand side of relation (2.10) in the weak sense, we obtain the following:

$$
\begin{aligned}
I \stackrel{\text { def }}{=} & \int_{-\infty}^{\infty} \omega^{k}\left(\frac{-x+\phi_{1}}{\varepsilon}\right) \omega^{\ell}\left(\frac{x-\phi_{2}}{\varepsilon}\right) \psi(x) d x \\
= & \int_{-\infty}^{\infty} \omega^{k}\left(\frac{-x+\phi_{1}}{\varepsilon}\right) \omega^{\ell}\left(\frac{x-\phi_{2}}{\varepsilon}\right) \frac{d}{d x} \int_{-\infty}^{x} \psi\left(x^{\prime}\right) d x^{\prime} d x \\
= & -\int_{-\infty}^{\infty} \psi_{0}(x)\left\{\omega^{\ell}\left(\frac{x-\phi_{2}}{\varepsilon}\right) \frac{\partial}{\partial x} \omega^{k}\left(\frac{-x+\phi_{1}}{\varepsilon}\right)+\omega^{k}\left(\frac{-x+\phi_{1}}{\varepsilon}\right) \frac{\partial}{\partial x} \omega^{\ell}\left(\frac{x-\phi_{2}}{\varepsilon}\right)\right\} d x \\
= & k \int_{-\infty}^{\infty} \omega^{k-1}(\eta) \omega_{\eta}^{\prime}(\eta) \omega^{\ell}(-\sigma-\eta) \psi_{0}\left(\phi_{1}-\varepsilon \eta\right) d \eta \\
& -\ell \int_{-\infty}^{\infty} \omega^{\ell-1}(\eta) \omega_{\eta}^{\prime}(\eta) \omega^{k}(-\sigma-\eta) \psi_{0}\left(\phi_{2}+\varepsilon \eta\right) d \eta,
\end{aligned}
$$

where $\psi_{0}(x)=\int_{-\infty}^{x} \psi\left(x^{\prime}\right) d x^{\prime}$, and $\psi \in \mathscr{D}\left(\mathbb{R}^{1}\right)$, and we took into account the exponential rate of vanishing of the product $\omega(\eta) \omega(-\eta)$ as $\eta \rightarrow \pm \infty$. Now, applying the Taylor expansion and using notation (2.10), we can rewrite the right-hand side in (2.13) in the following form:

$$
I=b_{k \ell} \psi_{0}\left(\phi_{1}\right)-b_{\ell k} \psi_{0}\left(\phi_{2}\right)-\varepsilon c_{k \ell} \psi\left(\phi_{1}\right)-\varepsilon c_{\ell k} \psi\left(\phi_{2}\right)+\mathcal{O}\left(\varepsilon^{2}\right)
$$

A detailed analysis of the integrals in (2.11) implies the following statement.

LEMma 2.3. The convolutions $b_{k e}$ and $c_{k \ell}$ exist and have the following properties:

$$
\begin{gathered}
b_{k \ell}(\sigma)=b_{\ell k}(\sigma)>0, \quad \frac{\sigma}{2} \frac{d b_{k k}}{d \sigma}+\frac{d c_{k k}}{d \sigma}=0, \\
\sigma \frac{d b_{12}}{d \sigma}+\frac{d}{d \sigma}\left(c_{12}+c_{21}\right)=0, \\
\lim _{\sigma \rightarrow+\infty} b_{k \ell}(\sigma)=\lim _{\sigma \rightarrow+\infty} c_{k \ell}(\sigma)=0, \quad \lim _{\sigma \rightarrow-\infty} b_{k \ell}=1, \quad \text { for any } k, \ell \geqslant 1, \\
\lim _{\sigma \rightarrow-\infty} c_{1 \ell}=0, \quad \lim _{\sigma \rightarrow-\infty} c_{2 \ell}=c_{2} \stackrel{\text { def }}{=} \int_{-\infty}^{\infty} \eta\left(\omega^{2}(\eta)\right)_{\eta}^{\prime} d \eta, \quad \text { for } \ell \geqslant 1 .
\end{gathered}
$$


3116 Interaction of shock waves in gas dynamics

Moreover,

$$
\begin{gathered}
\text { for } \ell \geqslant 1, k>1, \quad \lim _{\sigma \rightarrow+\infty} \frac{b_{\ell k}}{b_{11}^{\ell}}=0, \quad \lim _{\sigma \rightarrow+\infty} \frac{c_{11}}{b_{11}}=\alpha_{1} \sigma, \\
\text { for } i=1,2, \quad \lim _{\sigma \rightarrow+\infty} \frac{c_{i 2}}{b_{11}^{i}}=\beta_{i}, \quad \lim _{\sigma \rightarrow+\infty} \frac{c_{21}}{b_{11}}=0, \\
\text { for } j=1,2, \quad \lim _{\sigma \rightarrow+\infty} \sigma^{j-1}\left(\frac{d b_{1 j}}{d \sigma} / b_{11}\right)=\gamma_{j}, \quad \lim _{\sigma \rightarrow+\infty}\left(\frac{d c_{12}}{d \sigma} / b_{11}\right)=c,
\end{gathered}
$$

where $\alpha_{1}, \beta_{i}, \gamma_{j}$, and c are constants.

Using the first equation in (2.15) and the obvious relation $\psi_{0}\left(\phi_{i}\right)=\int_{-\infty}^{\infty} H(-x+$ $\left.\phi_{i}\right) \psi(x) d x$, we see that (2.14) implies the desired relation (2.10). This completes the proof of Lemma 2.2.

Applying the statement of Lemma 2.2, we obtain that the weak asymptotic of the anzatz (2.4) has the following form:

$$
\begin{aligned}
\rho_{\varepsilon}= & \rho_{0}-r b_{11}+\left(e_{1}+r b_{11}\right) H\left(-x+\phi_{1}\right)+\left(e_{2}+r b_{11}\right) H\left(x-\phi_{2}\right) \\
& -\operatorname{crc}_{11}\left(\delta\left(x-\phi_{1}\right)+\delta\left(x-\phi_{2}\right)\right)+O_{\mathscr{D}^{\prime}}\left(\varepsilon^{2}\right), \\
u_{\varepsilon}= & -v b_{11}+\left(u_{1}+v b_{11}\right) H\left(-x+\phi_{1}\right)+\left(u_{2}+v b_{11}\right) H\left(x-\phi_{2}\right)+O_{\mathscr{D}^{\prime}}\left(\varepsilon^{2}\right) .
\end{aligned}
$$

Therefore, for time after the interaction, we obtain two shock waves (or one wave if $\lim _{\tau \rightarrow-\infty}\left(\phi_{1}-\phi_{2}\right)=0$ ) with new amplitudes and new trajectories of motion. It is clear also that assumptions (2.8) are critical ones. Indeed, the breakdown of (2.8) implies the realization of a scenario of shock waves interaction which is qualitatively different from the stable scenario.

Now, let us find equations for the functions $\phi_{i}, r$, and $v$. To this end, we should calculate weak asymptotic expansions for the expressions in the integrals (2.1). Applying the statement of Lemmas 2.2 and 2.3 and using the notation

$$
V=v b_{11}, \quad R=r b_{11}, \quad B_{i k}=\frac{b_{i k}}{b_{11}^{i}}, \quad C_{i k}=\frac{c_{i k}}{b_{11}^{i}},
$$

after simple calculations, we obtain the following.

Lemma 2.4. Under the assumptions (2.2) and (2.3), the following relations hold:

$$
\begin{aligned}
\rho_{\varepsilon} u_{\varepsilon}= & \rho_{1} u_{1} H\left(-x+\phi_{1}\right)+\rho_{2} u_{2} H\left(x+\phi_{2}\right) \\
& +G_{1}\left\{H\left(-x+\phi_{1}\right)-H\left(-x+\phi_{2}\right)\right\} \\
& +\varepsilon G_{2} \delta\left(x-\phi_{1}\right)+\varepsilon G_{3} \delta\left(x-\phi_{2}\right)+O_{\mathscr{D}^{\prime}}\left(\varepsilon^{2}\right), \\
\rho_{\varepsilon} u_{\varepsilon}^{2}= & \rho_{1} u_{1}^{2} H\left(-x+\phi_{1}\right)+\rho_{2} u_{2}^{2} H\left(x+\phi_{2}\right) \\
& +G_{4}\left\{H\left(-x+\phi_{1}\right)-H\left(-x+\phi_{2}\right)\right\}+O_{\mathscr{D}^{\prime}}(\varepsilon),
\end{aligned}
$$


where

$$
\begin{aligned}
& G_{i}=g_{i 0}+g_{i 1} V+g_{i 2} R+B_{22} R V, \quad i=1,2,3, \\
& G_{4}=g_{40}+g_{41} V+g_{42} R+g_{43} V^{2}+g_{44} R V+B_{33} R V^{2}, \\
& g_{10}=\left(e_{1} u_{2}+e_{2} u_{1}\right) b_{11}, \quad g_{20}=g_{30}=\left(e_{1} u_{2}+e_{2} u_{1}\right) c_{11}, \\
& g_{11}=\rho_{0}+\left(e_{1}+e_{2}\right) B_{12}, \quad g_{12}=\left(u_{1}+u_{2}\right) B_{12}, \\
& g_{21}=\rho_{0} C_{11}+e_{1} C_{21}+e_{2} C_{12}, \quad g_{22}=u_{1} C_{21}+u_{2} C_{12}, \\
& g_{31}=\rho_{0} C_{11}+e_{2} C_{21}+e_{1} C_{12}, \quad g_{32}=u_{2} C_{21}+u_{1} C_{12}, \\
& g_{40}=2 \rho_{0} u_{1} u_{2} b_{11}+\left[\left(e_{1} u_{2}+e_{2} u_{1}\right)\left(u_{1}+u_{2}\right)+\left(e_{1}+e_{1}\right) u_{1} u_{2}\right] b_{12}, \\
& g_{41}=2\left[\rho_{0}\left(u_{1}+u_{2}\right)+\left(e_{1} u_{1}+e_{2} u_{2}\right) B_{13}+\left(e_{1} u_{2}+e_{2} u_{1}\right) b_{11} B_{22}\right], \\
& g_{42}=\left(u_{1}^{2}+u_{2}^{2}\right) B_{13}+2 u_{1} u_{2} b_{11} B_{22}, \quad g_{43}=\rho_{0} B_{22}+\left(e_{1}+e_{2}\right) B_{23}, \\
& g_{44}=2\left(u_{1}+u_{2}\right) B_{23} .
\end{aligned}
$$

Now, we should calculate the time derivatives. Since

$$
\frac{d \tau(t)}{d t}=\frac{\psi_{0_{t}}}{\varepsilon}, \quad \psi_{0_{t}}=\varphi_{20_{t}}-\varphi_{10_{t}}
$$

in order to obtain the precision $O(\varepsilon)$ in the right-hand side of relations $(2.1)$, we have to take into account the terms of order $O_{\mathscr{D}^{\prime}}(\varepsilon)$ in (2.18) and (2.21). At the same time, the phase derivatives do not include $O(1 / \mathcal{\varepsilon})$ terms since

$$
\frac{d \phi_{i}}{d t}=\varphi_{i 0_{t}}+\psi_{0_{t}} \varphi_{i 1}+\frac{\psi_{0}}{\varepsilon} \psi_{0_{t}} \varphi_{i 1}^{\prime}=\varphi_{i 0_{t}}+\psi_{0_{t}}\left(\tau \varphi_{i 1}\right)^{\prime}
$$

Here and in the sequel, the apostrophe denotes derivative with respect to $\tau$.

Next, using formulas (2.18), (2.25), and notation (2.20), we find that

$$
\begin{aligned}
\frac{\partial \rho_{\varepsilon}}{\partial t}= & \left(e_{1}+R\right) \frac{d \phi_{1}}{d t} \delta\left(x-\phi_{1}\right)-\left(e_{2}+R\right) \frac{d \phi_{2}}{d t} \delta\left(x-\phi_{2}\right) \\
& +\frac{\psi_{0 t}}{\varepsilon} R^{\prime}\left\{H\left(-x+\phi_{1}\right)-H\left(-x+\phi_{2}\right)\right\} \\
& -\psi_{0 t}\left(R C_{11}\right)^{\prime}\left\{\delta\left(x-\phi_{1}\right)-\delta\left(x-\phi_{2}\right)\right\}+O_{\mathscr{D}^{\prime}}(\varepsilon) .
\end{aligned}
$$

Now we need to use the following almost obvious statement.

Lemma 2.5. Let $S(\tau)$ be a function from the Schwartz space, and let a function $\phi_{k}(\tau) \in \mathscr{C}^{\infty}$ have the representation

$$
\phi_{k}(\tau)=x^{*}+\varepsilon \chi_{k}(\tau), \quad \chi_{k}(0)=0,
$$


3118 Interaction of shock waves in gas dynamics

where $x^{*}=$ const and $\chi_{k}$ is a slowly increasing function. Then

$$
S(\tau) H\left(-x+\phi_{k}(\tau)\right)=S(\tau) H\left(-x+x^{*}\right)+\varepsilon S(\tau) \chi_{k}(\tau) \delta\left(x-x^{*}\right)+\mathscr{O}_{\mathscr{D}^{\prime}}\left(\varepsilon^{2}\right) .
$$

Moreover,

$$
S(\tau) \delta\left(x-x^{*}\right)=S(\tau) \delta\left(x-\phi_{k}(\tau)\right)+\bigcirc_{\mathscr{D}^{\prime}}(\varepsilon)
$$

Denoting $x^{*}, t^{*}$ the point and time instant of interaction of the paths $x=\varphi_{i 0}(t), i=$ 1,2 , we obtain the equality

$$
\tau=\frac{\psi_{0}(t)}{\varepsilon}=\psi_{0_{t}} \frac{t-t^{*}}{\varepsilon}
$$

Thus, for functions of the form (2.5), we have

$$
\phi_{k}=x^{*}+\varepsilon \tau\left(\frac{\varphi_{k 0_{t}}}{\psi_{0_{t}}}+\varphi_{k 1}(\tau)\right) \stackrel{\text { def }}{=} x^{*}+\varepsilon \chi_{k}(\tau) .
$$

The assumptions for $r$ and the properties of the convolutions $b_{k \ell}, c_{k \ell}$ imply exponential rate of vanishing of the functions $R^{\prime}$ and $\left(R C_{11}\right)^{\prime}$. This and the statement of Lemma 2.5 imply the following relation:

$$
\begin{aligned}
\frac{1}{\varepsilon} R^{\prime}\left\{H\left(-x+\phi_{1}\right)-H\left(-x+\phi_{2}\right)\right\} & =R^{\prime}\left(\chi_{1}-\chi_{2}\right) \delta\left(x-x^{*}\right)+\bigcirc_{\mathscr{D}^{\prime}}(\varepsilon) \\
& =-\frac{\sigma}{2} R^{\prime}\left\{\delta\left(x-\phi_{1}\right)+\delta\left(x-\phi_{2}\right)\right\}+\mathscr{O}_{\mathscr{D}^{\prime}}(\varepsilon) .
\end{aligned}
$$

Therefore,

$$
\frac{\partial \rho_{\varepsilon}}{\partial t}=\left\{\left(e_{1}+R\right) \frac{d \phi_{1}}{d t}-\psi_{0_{t}} L_{0}\right\} \delta\left(x-\phi_{1}\right)-\left\{\left(e_{2}+R\right) \frac{d \phi_{2}}{d t}+\psi_{0_{t}} L_{0}\right\} \delta\left(x-\phi_{2}\right)+O_{\mathscr{D}^{\prime}}(\varepsilon),
$$

where

$$
L_{0}=\frac{\sigma}{2} R^{\prime}+\left(R C_{11}\right)^{\prime}
$$

It remains to use equality (2.15). Introduce the following notation:

$$
\begin{gathered}
k_{i j}=\frac{\sigma}{2} b_{i j}+c_{i j}, \quad \dot{B}_{11}=\frac{1}{b_{11}} \frac{d b_{11}}{d \sigma}, \\
K_{1 i}=\frac{k_{1 i}}{b_{11}}, \quad i=1,2, \quad K_{21}=\frac{k_{21}}{b_{11}}, \quad K_{22}=\frac{k_{22}}{b_{11}^{2}} .
\end{gathered}
$$

Then

$$
L_{0}=K_{11}\left(R^{\prime}-R \dot{B}_{11} \sigma^{\prime}\right)
$$


Preparing similar calculations for the time derivative of $\rho_{\varepsilon} \mathcal{u}_{\varepsilon}$, we obtain the formula

$$
\begin{aligned}
\frac{\partial \rho_{\varepsilon} u_{\varepsilon}}{\partial t}= & \left\{\left(\rho_{1} u_{1}+G_{1}\right) \frac{d \phi_{1}}{d t}-\psi_{0_{t}} L_{1}\right\} \delta\left(x-\phi_{1}\right) \\
& -\left\{\left(\rho_{2} u_{2}+G_{1}\right) \frac{d \phi_{2}}{d t}+\psi_{0_{t}} L_{2}\right\} \delta\left(x-\phi_{2}\right)+O_{\mathscr{D}^{\prime}}(\varepsilon),
\end{aligned}
$$

where

$$
\begin{aligned}
L_{i} & =\ell_{i 1}+\ell_{i 2} \sigma^{\prime}, \quad \ell_{i 1}=M_{i} V^{\prime}+N_{i} R^{\prime}, \quad i=1,2, \\
M_{1} & =\rho_{0} K_{11}+e_{2} K_{12}+e_{1} K_{21}+R K_{22}, \quad N_{1}=u_{2} K_{12}+u_{1} K_{21}+V K_{22}, \\
M_{2} & =\rho_{0} K_{11}+e_{1} K_{12}+e_{2} K_{21}+R K_{22}, \quad N_{2}=u_{1} K_{12}+u_{2} K_{21}+V K_{22}, \\
\ell_{12} & =\left\{\left(e_{2}-e_{1}\right) D_{12}-M_{1} \dot{B}_{11}\right\} V+\left\{\left(u_{2}-u_{1}\right) D_{12}-N_{1} \dot{B}_{11}\right\} R, \\
\ell_{22} & =-\left\{\left(e_{2}-e_{1}\right) D_{12}+M_{2} \dot{B}_{11}\right\} V-\left\{\left(u_{2}-u_{1}\right) D_{12}+N_{2} \dot{B}_{11}\right\} R, \\
D_{12} & =\frac{1}{b_{11}}\left\{\frac{\sigma}{2} \frac{d b_{12}}{d \sigma}+\frac{d c_{12}}{d \sigma}\right\} .
\end{aligned}
$$

Next, using formulas (2.18), (2.21), and (2.22), it is easy to calculate the derivatives

$$
\begin{gathered}
\frac{\partial \rho_{\varepsilon}}{\partial x}=-\left(e_{1}+R\right) \delta\left(x-\phi_{1}\right)+\left(e_{2}+R\right) \delta\left(x-\phi_{2}\right)+O_{\mathscr{D}^{\prime}}(\varepsilon), \\
\frac{\partial \rho_{\varepsilon} u_{\varepsilon}}{\partial x}=-\left(\rho_{1} u_{1}+G_{1}\right) \delta\left(x-\phi_{1}\right)+\left(\rho_{2} u_{2}+G_{1}\right) \delta\left(x-\phi_{2}\right)+O_{\mathscr{D}^{\prime}}(\varepsilon), \\
\frac{\partial \rho_{\varepsilon} u_{\varepsilon}^{2}}{\partial x}=-\left(\rho_{1} u_{1}^{2}+G_{4}\right) \delta\left(x-\phi_{1}\right)+\left(\rho_{2} u_{2}^{2}+G_{4}\right) \delta\left(x-\phi_{2}\right)+O_{\mathscr{D}^{\prime}}(\varepsilon) .
\end{gathered}
$$

Substituting expressions (2.32), (2.37), and (2.39) into relations (2.1), collecting coefficients of $\delta\left(x-\phi_{1}\right)$ and $\delta\left(x-\phi_{2}\right)$ and setting them equal to zero, we obtain

$$
\begin{aligned}
\left(e_{1}+R\right) \frac{d \phi_{1}}{d t} & =\psi_{0_{t}} L_{0}+G_{1}+\rho_{1} u_{1}, \\
\left(e_{2}+R\right) \frac{d \phi_{2}}{d t} & =-\psi_{0_{t}} L_{0}+G_{1}+\rho_{2} u_{2}, \\
\left(\rho_{1} u_{1}+G_{1}\right) \frac{d \phi_{1}}{d t} & =\psi_{0_{t}} L_{1}+G_{4}+\rho_{1} u_{1}^{2}+c_{0}^{2}\left(e_{1}+R\right), \\
\left(\rho_{2} u_{2}+G_{1}\right) \frac{d \phi_{2}}{d t} & =-\psi_{0_{t}} L_{2}+G_{4}+\rho_{2} u_{2}^{2}+c_{0}^{2}\left(e_{2}+R\right) .
\end{aligned}
$$

Theorem 2.6. Let there exist a smooth solution $R, V$, and $\phi_{i}, i=1,2$, of the system (2.40)(2.43) such that relations (2.7) and (2.8) hold. Then the weak asymptotic solution (2.4) describes the stable scenario of the shock waves interaction uniformly in time. 
3120 Interaction of shock waves in gas dynamics

Proof. To prove this statement, it is enough to consider system (2.40)-(2.43) for $\tau \rightarrow \pm \infty$. Let $\tau \rightarrow+\infty$. Assumption (2.7) and the vanishing of the convolutions imply the relations

$$
G_{i}, L_{i}, R, V \longrightarrow 0, \quad \frac{d \phi_{i}}{d t} \longrightarrow \varphi_{i 0_{t}}, \quad i=1,2, \text { as } \tau \longrightarrow+\infty .
$$

Thus, the system (2.40)-(2.43) transforms into the following:

$$
e_{i} \varphi_{i 0_{t}}=\rho_{i} u_{i}, \quad \rho_{i} u_{i} \varphi_{i 0_{t}}=\rho_{i} u_{i}^{2}+c_{0}^{2} \rho_{i}, \quad i=1,2 .
$$

Obviously, equalities (2.45) are the Rankine-Hugoniot conditions for the shock waves with amplitudes $\left(e_{i}, u_{i}\right), i=1,2$, which propagate over the unperturbed gas with the state $\left(\rho_{0}, u_{0}=0\right)$.

Now, let us consider system (2.40)-(2.43) for $\tau \rightarrow-\infty$, that is, for times after the interaction.

Assumptions (2.8) and stabilization of the convolutions imply the relations

$$
\phi_{i} \longrightarrow \bar{\phi}_{i}(t), \quad R \longrightarrow \bar{r}, \quad V \longrightarrow \bar{v} \quad \text { as } \tau \longrightarrow-\infty .
$$

Using the explicit formulas (2.23), it is easy to establish that

$$
G_{1} \longrightarrow \rho^{*} u^{*}-\rho_{1} u_{1}-\rho_{2} u_{2}, \quad G_{4} \longrightarrow \rho^{*} u^{* 2}-\rho_{1} u_{1}^{2}-\rho_{2} u_{2}^{2},
$$

where

$$
\rho^{*}=\rho_{0}+e_{1}+e_{2}+\bar{r}, \quad u^{*}=u_{1}+u_{2}+\bar{v}
$$

Therefore, the system (2.40)-(2.43) reduces to

$$
\begin{aligned}
\left(\rho^{*}-\rho_{2}\right) \bar{\phi}_{1_{t}} & =\rho^{*} u^{*}-\rho_{2} u_{2}, \\
\left(\rho^{*} u^{*}-\rho_{2} u_{2}\right) \bar{\phi}_{1_{t}} & =\rho^{*} u^{* 2}-\rho_{2} u_{2}^{2}+c_{0}^{2}\left(\rho^{*}-\rho_{2}\right), \\
\left(\rho^{*}-\rho_{1}\right) \bar{\phi}_{2_{t}} & =\rho^{*} u^{*}-\rho_{1} u_{1}, \\
\left(\rho^{*} u^{*}-\rho_{1} u_{1}\right) \bar{\phi}_{2_{t}} & =\rho^{*} u^{* 2}-\rho_{1} u_{1}^{2}+c_{0}^{2}\left(\rho^{*}-\rho_{1}\right) .
\end{aligned}
$$

Thus, we obtain the Rankine-Hugoniot conditions for two shock waves which propagate over the backgrounds $\left(\rho_{2}, u_{2}\right)$ and $\left(\rho_{1}, u_{1}\right)$, respectively. Obviously, equalities (2.49) imply the standard formulas for the limiting velocities of the front motions

$$
\bar{\phi}_{1_{t}}=u^{*}+c_{0} \sqrt{\frac{\rho_{2}}{\rho^{*}}}, \quad \bar{\phi}_{2_{t}}=u^{*}-c_{0} \sqrt{\frac{\rho_{1}}{\rho^{*}}} .
$$

Moreover, solving (2.49) for $\rho^{*}, u^{*}$, we find the expressions

$$
\rho^{*}=\frac{\rho_{1} \rho_{2}}{\rho_{0}}, \quad u^{*}=u_{1}+u_{2}
$$


which coincide with the well-known solution of the Riemann problem in the situation under consideration. Thus, we can treat the system (2.40)-(2.43) as a generalization of the Rankine-Hugoniot conditions for two interacting shocks with opposite directions of motion.

\section{Investigation of the dynamical system}

First of all, let us pass from (2.40)-(2.43) to a system of three autonomous equations. To do this, let us solve (2.40), (2.41) with respect to $\phi_{1_{t}}, \phi_{2_{t}}$ and subtract one from the other. Since

$$
\frac{d \phi_{2}}{d t}-\frac{d \phi_{1}}{d t}=\psi_{0_{t}} \frac{d}{d \tau} \frac{\psi_{2}-\phi_{1}}{\varepsilon}=\psi_{0_{t}} \frac{d \sigma}{d \tau}
$$

we obtain the equality

$$
\frac{d \sigma}{d \tau}=\frac{1}{\psi_{0_{t}}}\left\{\frac{G_{1}+\rho_{2} u_{2}}{e_{2}+R}-\frac{G_{1}+\rho_{1} u_{1}}{e_{1}+R}\right\}-L_{0}\left\{\frac{1}{e_{2}+R}+\frac{1}{e_{1}+R}\right\}
$$

Next, let us note that (2.40)-(2.43) imply the following compatibility conditions:

$$
\frac{(-1)^{k+1} \psi_{0_{t}} L_{0}+G_{1}+\rho_{k} u_{k}}{e_{k}+R}=\frac{(-1)^{k+1} \psi_{0_{t}} L_{0}+G_{4}+\rho_{k} u_{k}^{2}+c_{0}^{2}\left(e_{k}+R\right)}{\rho_{k} u_{k}+G_{1}},
$$

for $k=1,2$. Now, solving (3.2), (3.3) with respect to the derivatives $\sigma_{\tau}{ }^{\prime}, R_{\tau}{ }^{\prime}, V_{\tau}{ }^{\prime}$, we can rewrite these equations in the standard form

$$
\psi_{0_{t}} \frac{d U}{d \tau}=F(U), \quad U=(\sigma, R, V)
$$

Obviously, assumptions (2.7) imply the following scattering-type conditions:

$$
\frac{\sigma}{\tau} \longrightarrow 1, \quad R \longrightarrow 0, \quad V \longrightarrow 0 \quad \text { as } \tau \longrightarrow \infty
$$

Our aim is to establish the existence of a global solution for the problem (3.4), (3.5), and to discover the behavior of $\sigma, R$, and $V$ for $\tau \rightarrow-\infty$. However, the explicit formulas for the right-hand side $F$ are rather unwieldy. In order to avoid too complicated algebraic calculations, we restrict ourselves to the special case $\rho_{1}=\rho_{2}$. It is easy to establish that this choice implies the equality $u_{1}=-u_{2}$, and moreover, $V \equiv 0$. Thus, in the special case, we pass from the system (3.4) to the following system of two equations:

$$
\begin{aligned}
& \psi_{0_{t}} \frac{d \sigma}{d \tau}=4 u_{1} \frac{\alpha_{2}+q_{1} K_{11} R}{\alpha_{3}+\alpha_{4} R} \stackrel{\text { def }}{=} F_{1}, \\
& \psi_{0_{t}} \frac{d R}{d \tau}=2 u_{1} \frac{e_{1} q_{0}+\left(q_{0}-\alpha_{0}\right) R-\alpha_{1} R^{2}}{\alpha_{3}+\alpha_{4} R} \stackrel{\text { def }}{=} F_{2},
\end{aligned}
$$


3122 Interaction of shock waves in gas dynamics

where $\psi_{0_{t}}=-2 u_{1} \rho_{1} / e_{1}$, and

$$
\begin{aligned}
& q_{0}=\rho_{0} b_{11}+e_{1} b_{12}, \quad q_{1}=\frac{\rho_{0} \rho_{1}}{2 e_{1}^{2}}+B_{13}-b_{11} B_{22}, \\
& \alpha_{0}=\frac{\rho_{1}^{2}}{2 e_{1}}+e_{1} q_{1}+2 \rho_{1} D_{12}+\dot{B}_{11}\left(\rho_{1} N+2 K_{11}\left(q_{0}-\frac{\rho_{1}^{2}}{2 e_{1}}\right)\right), \\
& \alpha_{1}=q_{1}\left(1-2 K_{11} \dot{B}_{11}\right), \quad \alpha_{2}=K_{11}\left(\frac{\rho_{1}^{2}}{2 e_{1}}-q_{0}\right)-\frac{\rho_{1} N}{2}, \\
& \alpha_{3}=e_{1} N-\rho_{1} K_{11}, \quad \alpha_{4}=N+4 K_{11} D_{12}, \quad N=K_{21}-K_{12} .
\end{aligned}
$$

There are four curves that specify the behavior of the system (3.6) trajectories. Let us denote

$$
\gamma_{1}^{ \pm}=\left\{R=R_{1}^{ \pm}(\sigma), \sigma \in \mathbb{R}^{1}\right\}, \quad R_{1}^{ \pm}=\frac{1}{2 \alpha_{1}}\left(q_{0}-\alpha_{0} \pm \sqrt{\left(\alpha_{0}-q_{0}\right)^{2}+4 \alpha_{1} e_{1} q_{0}}\right),
$$

the isoclines of $F_{1}$,

$$
\gamma_{2}=\left\{R=R_{2}(\sigma), \sigma \in \mathbb{R}^{1}\right\}, \quad R_{2}=-\frac{\alpha_{2}}{q_{1} K_{11}},
$$

the isocline of $F_{2}$, and

$$
\gamma_{s}=\left\{R=R_{3}(\sigma), \sigma \in \mathbb{R}^{1}\right\}, \quad R_{s}=-\frac{\alpha_{3}}{\alpha_{4}},
$$

the curve of singularities.

The statement of Lemma 2.3 implies the following relations:

$$
\begin{gathered}
N \longrightarrow c_{2}, \quad K_{11} \longrightarrow \frac{\sigma}{2}, \quad D_{12}, \dot{B}_{11} \longrightarrow 0, \quad q_{0} \longrightarrow \rho_{1}, \quad q_{1}, \alpha_{1} \longrightarrow \frac{\rho_{0} \rho_{1}}{2 e_{1}^{2}}, \\
\alpha_{0} \longrightarrow \frac{\rho_{1}\left(\rho_{0}+\rho_{1}\right)}{2 e_{1}}, \quad \alpha_{2} \longrightarrow-\frac{\rho_{1}\left(\sigma\left(\rho_{1}-2 \rho_{0}\right)+2 c_{2} e_{1}\right)}{4 e_{1}}, \\
\alpha_{3}=-\frac{\sigma \rho_{1}}{2}+e_{1} c_{2}, \quad \alpha_{4} \longrightarrow c_{2} \quad \text { as } \sigma \longrightarrow-\infty .
\end{gathered}
$$

Therefore, for $\sigma \rightarrow-\infty$,

$$
R_{1}^{+} \longrightarrow \frac{e_{1}^{2}}{\rho_{0}}, \quad R_{1}^{-} \longrightarrow-2 e_{1}, \quad R_{2} \longrightarrow-\frac{e_{1}}{\rho_{0}}\left(\rho_{1}-2 e_{1}\right), \quad R_{s} \longrightarrow \sigma \frac{\rho_{1}}{2 c_{2}} .
$$

To consider the behavior of the curves $\gamma$ for $\sigma \rightarrow+\infty$, we should make more precise estimates of the convolutions. Indeed, with the accuracy of Lemma 2.3, we obtain the relations $\alpha_{1} \rightarrow 0, \alpha_{4} \rightarrow 0$ as $\sigma \rightarrow+\infty$ and loose the signs of the curves. The simplest way to overcome this difficulty is to note that the limiting Rankine-Hugoniot conditions (2.45) and (2.49) do not depend on the choice of the regularization $\omega$. Thus we can use a specific 
regularization, for instance, $\omega(\eta)=(1+\tanh \eta) / 2$. For such a choice, we find

$$
\begin{gathered}
N \longrightarrow \frac{1}{2}, \quad K_{11} \longrightarrow-\frac{\sigma}{2(2 \sigma-1)}, \quad D_{12} \longrightarrow \frac{\sigma-1}{2 \sigma-1}, \\
\dot{B}_{11} \longrightarrow-4 \frac{\sigma-1}{2 \sigma-1}, \quad q_{0} \longrightarrow 0, \quad q_{1} \longrightarrow \frac{\rho_{0} \rho_{1}}{2 e_{1}^{2}}, \\
\alpha_{0} \longrightarrow e_{1} q_{1}, \quad \alpha_{1} \longrightarrow \frac{q_{1}}{4 \sigma^{2}}, \quad \alpha_{2} \longrightarrow-\frac{\rho_{1}\left(\rho_{1}+2 e_{1}\right)}{8 e_{1}}, \\
\alpha_{3} \longrightarrow \frac{1}{4}\left(\rho_{1}+2 e_{1}\right), \quad \alpha_{4} \longrightarrow \frac{1}{8 \sigma^{2}} \quad \text { as } \sigma \longrightarrow \infty .
\end{gathered}
$$

Thus,

$$
R_{1}^{+} \longrightarrow 0, \quad R_{1}^{-} \longrightarrow-4 \sigma^{2} e_{1}, \quad R_{2} \longrightarrow-\frac{e_{1}}{\rho_{0}}\left(\rho_{1}+2 e_{1}\right), \quad R_{s} \longrightarrow-2 \sigma^{2}\left(\rho_{1}+2 e_{1}\right)
$$

Numerical simulations show that the curves $\gamma_{1}^{ \pm}, \gamma_{2}$, and $\gamma_{s}$ do not intersect for finite $\sigma$. This implies that the system (3.6) does not have any critical points. At the same time, the parts of the curve $\gamma_{1}^{+}$for $\sigma \rightarrow \pm \infty$ play the role of a saddle point and an attractive node, respectively. Indeed, let $\sigma \rightarrow-\infty$ and $R=e_{1}^{2} / \rho_{0}+r$. Then, formulas (3.11) imply the following linearization of system (3.6):

$$
\frac{d \sigma}{d \tau}=\frac{\rho_{0}}{\rho_{1}}\left(1+\frac{r}{e_{1}}\right), \quad \frac{d r}{d \tau}=-\frac{\rho_{0}+\rho_{1}}{\rho_{1}} \frac{r}{\sigma} \quad \text { for } \sigma \ll-1
$$

The solution $r=r(\sigma)$ has the form

$$
e_{1} \ln \left(|r||\sigma|^{\beta}\right)+r=\text { const, } \quad \beta=\frac{\left(\rho_{0}+\rho_{1}\right)}{\rho_{0}}>0
$$

So, in the leading term

$$
\sigma=\frac{\rho_{0}}{\rho_{1}} \tau, \quad|r|=|c \sigma|^{-\beta}, \quad c=\text { const }
$$

and it is clear that the line $R=e_{1}^{2} / \rho_{0}, \sigma=\rho_{0} \tau / \rho_{1}$ for $\tau \rightarrow-\infty$ is similar to an attractive node.

Let $\sigma \rightarrow \infty$. Formulas (3.13) imply the following linearization of system (3.6):

$$
\frac{d \sigma}{d \tau}=\left(1+\frac{\beta_{1}}{e_{1}} R\right), \quad \frac{d R}{d \tau}=2 \beta_{1} R, \quad \beta_{1}=\frac{\rho_{0}}{\rho_{1}+2 e_{1}}>0
$$

So, the trajectories go out a neighborhood of the line $\sigma=\tau, R=0$ with an exponential velocity, whereas $\sigma=\tau$ and $R=0$ satisfy system (3.6) for $\tau \rightarrow+\infty$. 
3124 Interaction of shock waves in gas dynamics

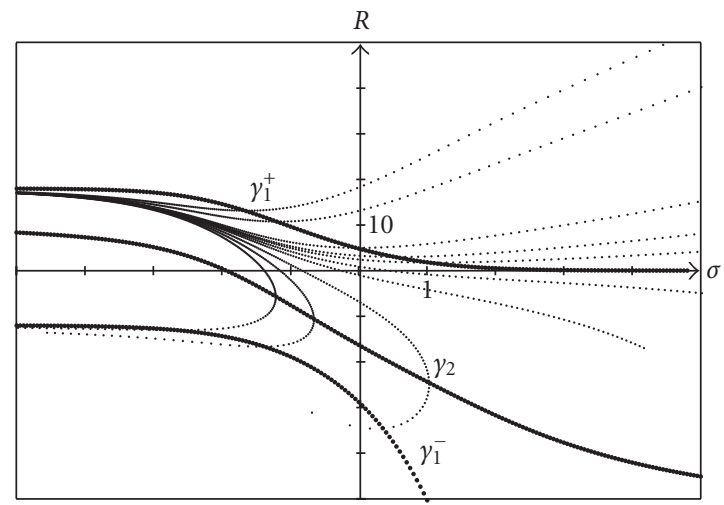

Figure 3.1. The phase portrait for system (3.6).

Therefore, taking into account the signs of the right-hand sides $F_{1}$ and $F_{2}$, we obtain the phase portrait shown in Figure 3.1.

Now, it is evident the existence of a separatrix which goes from $\bar{\sigma}_{-}=\rho_{0} \tau / \rho_{1}, \bar{R}_{-}=e_{1}^{2} / \rho_{0}$ (for $\tau \rightarrow-\infty$ ) to $\bar{\sigma}_{+}=\tau, \bar{R}_{+}=0$ (for $\tau \rightarrow \infty$ ) lying under the isocline $\gamma_{1}^{+}$. This implies the following statement.

THEOREM 3.1. There exists the separatrix for system (3.6) which coincides with the "points" $\bar{\sigma}_{-}, \bar{R}_{-}$and $\bar{\sigma}_{+}, \bar{R}_{+}$. This separatrix can be specified by the scattering-type conditions

$$
\frac{\sigma}{\tau} \longrightarrow 1, \quad R \longrightarrow 0 \quad \text { for } \tau \longrightarrow+\infty
$$

It seems that a similar statement is true in the general case of arbitrary $\rho_{1}$ and $\rho_{2}$. In any case, taking into account the limiting values of the convolutions, after cumbersome calculations, it is possible to find the limiting values of the functions

$$
\begin{gathered}
\bar{\sigma}_{-}=\tau \frac{\rho_{0}}{\sqrt{\rho_{1} \rho_{2}}}, \quad \bar{R}_{-}=\frac{e_{1} e_{2}}{\rho_{0}}, \quad \bar{V}_{+}=0 \quad \text { as } \tau \longrightarrow-\infty, \\
\bar{\sigma}_{+}=\tau, \quad \bar{R}_{+}=0, \quad \bar{V}_{-}=0 \quad \text { as } \tau \longrightarrow+\infty .
\end{gathered}
$$

\section{Calculations of the phase corrections}

After solving problem (3.4), (3.5), we can find the phase corrections $\varphi_{i 1}$. Using again the formulas (2.25), we can rewrite (2.40), (2.41) in the following form:

$$
\psi_{0_{t}} \frac{d}{d \tau}\left(\tau \varphi_{i 1}\right)=\frac{G_{1}+\rho_{i} u_{i}+(-1)^{i+1} \psi_{0_{t}} L_{0}}{e_{i}+R}-\varphi_{i 0_{t}} \stackrel{\text { def }}{=} f_{i}(U),
$$

where $i=1,2$, and $U=(\sigma, R, V)$.

Now, we readily derive the desired formulas as follows:

$$
\varphi_{i 1}(\tau)=\frac{1}{\psi_{0_{t}}} \frac{1}{\tau} \int_{0}^{\tau} f_{i}(U) d \tau^{\prime}, \quad i=1,2 .
$$


Smoothness of $U$ implies the boundedness of $f_{i}$ at the point $\tau=0$. Thus, $\varphi_{i 1}$ are bounded at this point. Next, since

$$
G_{1}, L_{0}, R \longrightarrow 0 \quad \text { as } \tau \longrightarrow+\infty, \quad \varphi_{i 0_{t}}=\frac{\rho_{i} u_{i}}{e_{i}}, \quad i=1,2,
$$

the functions $f_{i}$ vanish sufficiently rapidly as $\tau \rightarrow \infty$. This guarantees the convergence of the integral in the right-hand sides of (4.2) as $\tau \rightarrow \infty$. Hence,

$$
\varphi_{i 1}(\tau) \longrightarrow 0 \text { as } \tau \longrightarrow+\infty,
$$

which confirms the first a priori assumption in (2.7). Furthermore,

$$
f_{i}(\tau)=\frac{\rho^{*} u^{*}-\rho_{\bar{i}}^{\bar{u}} u_{\bar{i}}}{\rho^{*}-\rho_{\bar{i}}}-\varphi_{i 0_{t}}+\mathcal{O}\left(\tau^{2} e^{\gamma \tau}\right) \quad \text { as } \tau \longrightarrow+\infty,
$$

where we use the notation (2.48) and $\bar{r}=\bar{R}_{-}, \bar{v}=\bar{V}_{-}$since $B_{11} \rightarrow 1, \bar{i}=2$ for $i=1$, and $\bar{i}=1$ for $i=2$, and $\gamma$ is a number defined by the choice of the regularization $\omega$.

Thus, the integral diverges. By using L'Hospital rule, it is easy to find the limiting value of $\varphi_{i 1}$ as follows:

$$
\bar{\varphi}_{i 1}=\frac{1}{\psi_{0_{t}}}\left\{\frac{\rho^{*} u^{*}-\rho_{\bar{i}} u_{\bar{i}}}{\rho^{*}-\rho_{\bar{i}}}-\varphi_{i 0_{t}}\right\}, \quad i=1,2 .
$$

This satisfies the first a priori assumption in (2.13). Moreover, formulas (4.6) allow to calculate the limiting phases $\bar{\phi}_{i}=\lim _{\tau \rightarrow-\infty} \phi_{i}$. Indeed, using the Taylor expansion at the time instant $t=t^{*}$ and taking into account equality (2.30), we derive

$$
\begin{gathered}
\bar{\phi}_{i} \stackrel{\text { def }}{=} \varphi_{i 0}+\psi_{0}(t) \bar{\varphi}_{i 1}=x^{*}+\left(\varphi_{i 0_{t}}+\psi_{0_{t}} \bar{\varphi}_{i 1}\right)\left(t-t^{*}\right) \\
=x^{*}+\frac{\rho^{*} u^{*}-\rho_{\bar{i}} u_{\bar{i}}}{\rho^{*}-\rho_{\bar{i}}}\left(t-t^{*}\right), \quad i=1,2 .
\end{gathered}
$$

Obviously, these phases satisfy the first Rankine-Hugoniot conditions (2.49).

\section{Conclusion}

We considered the gas dynamics system as the most important example of hyperbolic systems of conservation laws. It seems obvious that the above described method of construction of uniform in time asymptotics can be applied to general strictly hyperbolic systems of conservation laws. Of course, the direct application of the method is possible only when interaction of shock waves results in the appearance of new shock waves. It is clear also that for systems of $n$ equations, $n>2$, the anzatz has to include all the possible stable shock waves. In such a case, sufficiently simple algebraic calculations allow to obtain a result similar to Theorem 2.6. However, the investigation of the corresponding dynamical system is not so easy in the general case. This and the appearance of centered rarefaction, contact discontinuities, and vacuum state are the issues for future investigation. 


\section{Acknowledgment}

The research was supported by SEP-CONACYT under Grant $41421 \mathrm{~F}$ and by CONACYT under Grant 43208 (Mexico).

\section{References}

[1] A. Bressan, Hyperbolic systems of conservation laws in one space dimension, Proceedings of the International Congress of Mathematicians, vol. I (Beijing, 2002), Higher Education Press, Beijing, 2002, pp. 159-178.

[2] C. M. Dafermos, Hyperbolic Conservation Laws in Continuum Physics, Grundlehren der mathematischen Wissenschaften, vol. 325, Springer, Berlin, 2000.

[3] V. G. Danilov, Generalized solutions describing singularity interaction, Int. J. Math. Math. Sci. 29 (2002), no. 8, 481-494.

[4] V. G. Danilov, G. A. Omel'yanov, and V. M. Shelkovich, Weak asymptotics method and interaction of nonlinear waves, Asymptotic Methods for Wave and Quantum Problems, Amer. Math. Soc. Transl. Ser. 2, vol. 208, American Mathematical Society, Rhode Island, 2003, pp. 33-163.

[5] V. G. Danilov and V. M. Shelkovich, Propagation and interaction of shock waves of quasilinear equation, Nonlinear Stud. 8 (2001), no. 1, 135-169.

[6] Delta-shock wave type solution of hyperbolic systems of conservation laws, Quart. Appl. Math. 63 (2005), no. 3, 401-427.

[7] D. A. Kulagin and G. A. Omel'yanov, Asymptotics of kink-kink interaction for equations of sineGordon type, Mat. Zametki 75 (2004), no. 4, 603-607 (Russian), translated in Math. Notes 75 (2004), no. 3-4, 563-567.

[8] P. G. LeFloch, Hyperbolic Systems of Conservation Laws. The Theory of Classical and Nonclassical Shock Waves, Lectures in Mathematics ETH Zürich, Birkhäuser, Basel, 2002.

[9] B. L. Rozhdestvenskii and N. N. Yanenko, Systems of Quasilinear Equations and Their Applications to Gas Dynamics, 2nd ed., Nauka, Moscow, 1978, English translation, American Mathematical Society, Rhode Island, 1983.

[10] G. B. Whitham, Linear and Nonlinear Waves, Pure and Applied Mathematics, John Wiley \& Sons, New York, 1974.

M. G. García-Alvarado: Departamento de Matemáticas, Universidad de Sonora, 83000 Sonora, Mexico

E-mail address: mggarcia@gauss.mat.uson.mx

R. Flores-Espinoza: Departamento de Matemáticas, Universidad de Sonora, 83000 Sonora, Mexico

E-mail address: rflorese@gauss.mat.uson.mx

G. A. Omel'yanov: Departamento de Matemáticas, Universidad de Sonora, 83000 Sonora, Mexico

E-mail address: omel@hades.mat.uson.mx 


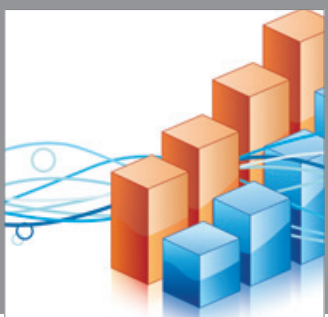

Advances in

Operations Research

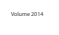

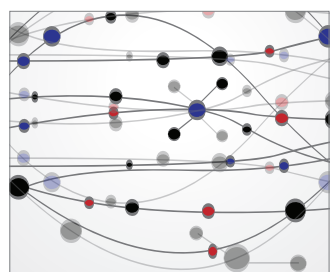

\section{The Scientific} World Journal
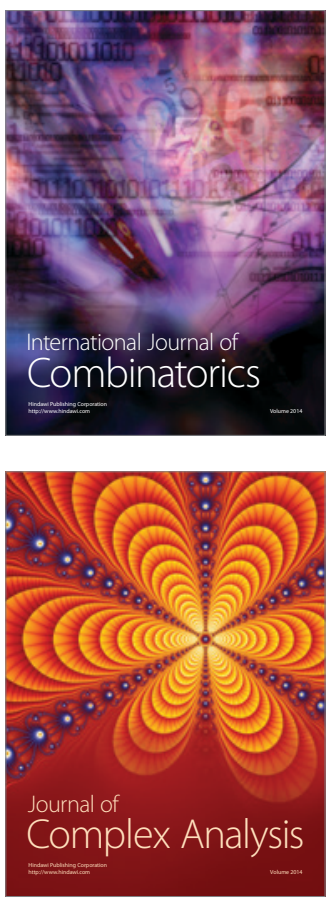

International Journal of

Mathematics and

Mathematical

Sciences
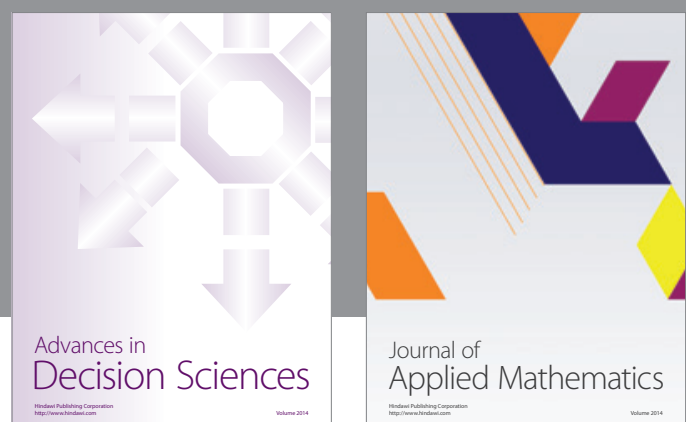

Journal of

Applied Mathematics
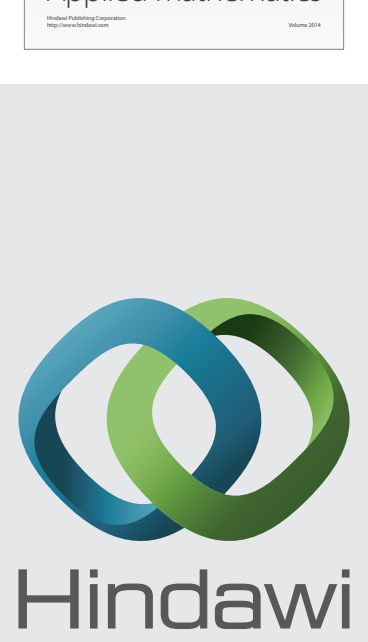

Submit your manuscripts at http://www.hindawi.com
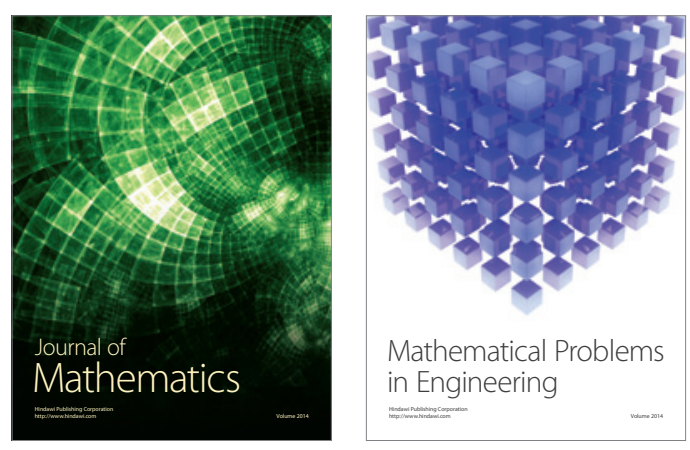

Mathematical Problems in Engineering
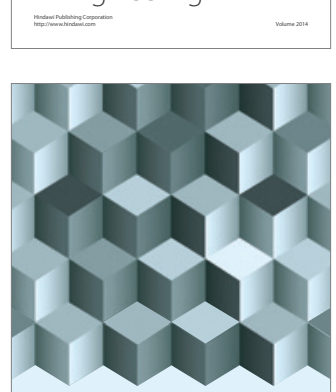

Journal of

Function Spaces
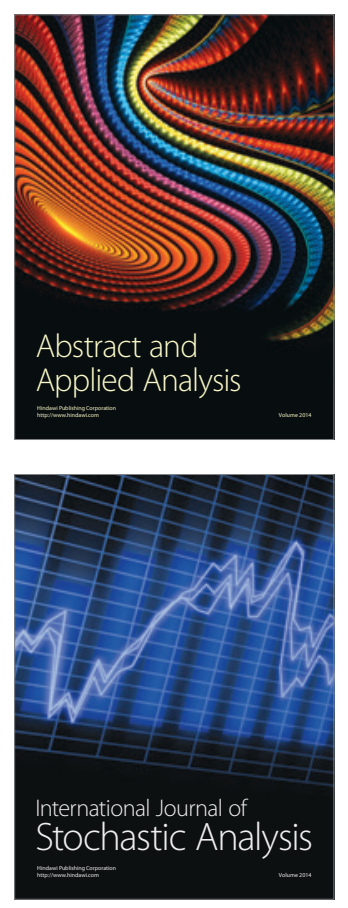

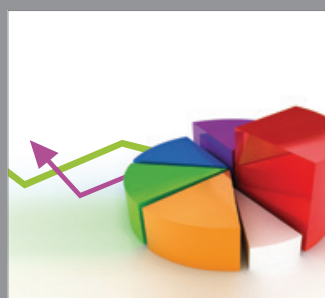

ournal of

Probability and Statistics

Promensencen
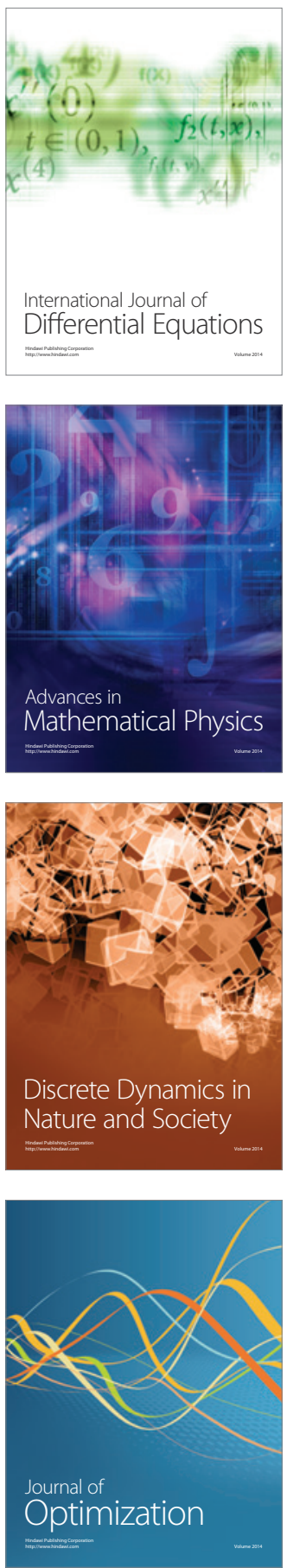